\title{
PAKC: A novel Panel of HLA class I Antigen presentation machinery Knockout Cells
} from the same genetic origin ${ }^{1}$

Antonius A. de Waard ${ }^{*}, t, 1$, Tamara Verkerk ${ }^{*}, t, 1$ Marlieke L.M. Jongsma ${ }^{*},+$, , Kelly Hoefakker ${ }^{*}, t$, Sunesh Sethumadhavan ${ }^{\S}$, Carolin Gerkef, $\|, \#, "$, Sophie Bliss",t, George M.C. Janssen ${ }^{\dagger \dagger}$, Arnoud H. de Ru ${ }^{\dagger \dagger}$, Frans H.J. Claas ${ }^{\ddagger \ddagger}$, Arend Mulder ${ }^{\ddagger \ddagger}$, Robert Tampés, Peter A. van Veelen ${ }^{\dagger \dagger}$, Anne Halenius ${ }^{\text {I. }} \|$, Robbert M. Spaapen ${ }^{*}, t$

"Department of Immunopathology, Sanquin Research, Amsterdam, The Netherlands

† Landsteiner Laboratory, Amsterdam UMC, University of Amsterdam, Amsterdam, The Netherlands

‡Oncode Institute and Department of Cell and Chemical Biology, LUMC, Leiden, The Netherlands

$\S$ Institute of Biochemistry, Biocenter, Goethe University Frankfurt, Frankfurt a.M., Germany

"Institute of Virology, Medical Center University of Freiburg, Freiburg, Germany

"Faculty of Medicine, University of Freiburg, Freiburg, Germany

\#Spemann Graduate School of Biology and medicine, University of Freiburg, Freiburg,

Germany

"Faculty of Biology, University of Freiburg, Freiburg, Germany

${ }^{\text {tt }}$ Center for Proteomics and Metabolomics, LUMC, Leiden, The Netherlands

\# Department of Immunohematology and Blood Transfusion, LUMC, Leiden, The

Netherlands

${ }^{1}$ These authors contributed equally to this work

Running title: Panel of Antigen presentation machinery Knockout Cells

Keywords: Human, Antigens/Peptides/Epitopes, MHC, HLA class I, antigen processing and presentation, CRISPR/Cas9, knockout,

Corresponding Author: Robbert Spaapen, PhD, Sanquin Research, Department of Immunopathology, Plesmanlaan 125, 1066CX, Amsterdam, The Netherlands. Phone: +31 610906464; e-mail: r.spaapen@sanquin.nl

\footnotetext{
${ }^{1}$ This work was supported by the Netherlands organization for scientific research (NWO-VENI 016.131.047; R.M.S.), KWF Alpe d'HuZes (Bas Mulder Award 2015-7982; R.M.S.), the Landsteiner Foundation for Blood Transfusion Research (LSBR fellowship 1842F; R.M.S.), the German Research Foundation (SFB 807 - Membrane Transport and Communication; R.T.) and an ERC Advanced Grant (EditMHC; R.T.).

Abbreviations used in this article: APM, antigen presentation machinery; B2M, beta-2 microglobulin; CALR, calreticulin; CNX, calnexin; HLA-I, HLA class I; PAKC, Panel of APM Knockout Cells; PLC, peptide loading complex.
} 
bioRxiv preprint doi: https://doi.org/10.1101/2020.01.24.917807; this version posted January 25,2020 . The copyright holder for this

preprint (which was not certified by peer review) is the author/funder, who has granted bioRxiv a license to display the preprint in perpetuity. It is made available under aCC-BY-NC 4.0 International license.

31

32

33

\section{Key points}

1. We generated a panel of cell lines to study HLA class I antigen presentation

2. We show how this will spark research in infection, tumor biology and autoimmunity 


\section{Abstract}

With the emergence of immunotherapies, the understanding of functional HLA class I antigen presentation to $T$ cells is more relevant than ever. Current knowledge on antigen presentation is based on decades of research in a wide variety of cell types with varying antigen presentation machinery (APM) expression patterns, proteomes and HLA haplotypes. This diversity complicates the establishment of individual APM contributions to antigen generation, selection and presentation. Therefore, we generated a novel Panel of APM Knockout Cell lines (PAKC) from the same genetic origin. After CRISPR/Cas9 genome-editing of ten individual APM components in a human cell line, we derived clonal cell lines and confirmed their knockout status and phenotype. We then show how PAKC will accelerate research on the functional interplay between APM components and their role in antigen generation and presentation. This will lead to improved understanding of peptide-specific $\mathrm{T}$ cell responses in infection, cancer and autoimmunity. 


\section{Introduction}

HLA class I (HLA-I) proteins are crucial for the onset of $T$ cell responses during infections, cancer or autoimmunity(1-3). HLA-I continuously samples peptides from intracellularly degraded proteins and presents these on the cell surface to $C D 8^{+} \mathrm{T}$ cells(3). To escape $\mathrm{T}$ cell surveillance, various viruses express inhibitors of the machinery required for antigen processing and loading onto HLA-I (antigen presentation machinery; APM)(2). For the same reason, tumor cells acquire genetic impairments in the APM, especially after immunotherapy(4). On the other hand, HLA-I alleles and functional polymorphisms in the APM have been highly associated with the development of several auto-inflammatory diseases(1). In the past decades, the individual function of each of the APM components has been widely studied(3). These studies revealed that newly synthesized HLA-I heavy chains are $N$ glycosylated and consecutively bound by the lectin chaperones calnexin (CNX) and calreticulin (CALR). Several glycosidases, such as glucosidase Ila, trim the glycan tree generating the core substrate for complex $\mathrm{N}$-glycosylation later on in the Golgi. After recruiting beta-2 microglobulin (B2M), the HLA-I heavy chain associates with the peptide loading complex consisting of ERp57, tapasin and the transporter associated with peptide loading (TAP1/TAP2 heterodimer)(5). The TAP heterodimer transports peptides derived from degraded proteins from the cytosol into the ER lumen, where they will be occasionally trimmed by the aminopeptidase ERAP1. Tapasin and ERp57 assist in the binding of peptides into the so-called HLA-I peptide binding groove, after which the mature HLA-I heterotrimer will be shuttled via the Golgi to the plasma membrane to present its peptide to $C D 8^{+} \mathrm{T}$ cells(6).

These studies were conducted using various (sets of) murine or human model cell lines with varying APM expression patterns, proteomes and HLA haplotypes, complicating the comparison of their individual contributions to antigen processing and presentation. Moreover, the molecular interplay between the different APM components may have confounding effects in assays with single APM component mutant cell lines. Previous efforts have established 721 lymphoblastic cell lines with gamma irradiation-induced TAP1, tapasin and HLA-I mutants, which have been used extensively to enlarge the knowledge on these factors. To address the current questions in the field, it is essential to generate a broader and more defined model system capable of overcoming these issues and addressing the current questions in the field. Here, we generated a novel panel of HLA-I APM knockout cell lines, designated PAKC, on the background of human HAP1 cells. Using CRISPR/Cas9, we generated ten clonal cell lines knocked out for individual components of the HLA-I antigen processing and presentation pathway. We illustrate how the availability of this collection to the community will spark stateof-the-art research to explain associations of the APM and certain HLA-I alleles with diseases. Additionally, we show the opportunities of PAKC to improve the rules of peptide processing 
bioRxiv preprint doi: https://doi.org/10.1101/2020.01.24.917807; this version posted January 25,2020 . The copyright holder for this preprint (which was not certified by peer review) is the author/funder, who has granted bioRxiv a license to display the preprint in perpetuity. It is made available under aCC-BY-NC 4.0 International license.

83 and presentation which will further increase our understanding of infection, cancer and 84 autoimmunity. 


\section{Materials and Methods}

\section{Cell culture}

HAP1 and HEK293T cell lines were cultured at $37{ }^{\circ} \mathrm{C}$ and $5 \% \mathrm{CO}_{2}$ in IMDM (Gibco) supplemented with 10\% FCS and antibiotics (PenStrep; Invitrogen). CD8 ${ }^{+}$T cell clones recognizing peptides derived from the endogenously expressed proteins USP11 and SSR1(7, 8) were expanded using a standard feeder mix in IMDM supplemented with 5\% human serum (Sanquin) and 5\% FCS(9).

\section{Genome editing and overexpression}

gRNAs used for gene editing are listed in Supplementary Table I. pX458 containing a B2M targeting gRNA was kindly provided by Dr. R. Mezzadra. ERp57 and tapasin targeting gRNAs in pX330 were co-transfected with a Blasticidin S-resistance overexpression vector as previously described(10). A gRNA targeting a conserved region in HLA-A, -B, $-C$ and $-G$ in a pX330 vector was transfected into HAP1 cells using X-tremeGENE (Roche). Single cells were FACS sorted based on W6/32 negativity to obtain knockout clones for HLA-A, -B and -C. gRNAs targeting CNX, ERAP1, CALR and glucosidase Ila in pLentiCRISPRv2 and a gRNA targeting Tap1 in pL.CRISPR.efs.GFP were co-transfected with packaging plasmids psPAX2 and pVSVg, and pAdVAntage (Promega) using polyethylenimine (PEI; Polyscience) into HEK293T cells for virus production. puc2CL6IP HLA-B*27:05/09 constructs (kind gift of Dr. S. Springer(11)) were co-transfected using PEI with packaging plasmids $\mathrm{pVSVg}$ and NLBH into HEK293T cells for virus production. Viral supernatants of other HLA alleles contained a $\triangle$ NGFR marker gene (kind gift of Dr. M Griffioen) (12). Viral supernatant was filtered and used for transduction by spinoculation in the presence of $8 \mu \mathrm{g} / \mathrm{mL}$ protamine sulfate. Several genome-edited cells were selected using puromycin or blasticidin $\mathrm{S}$, before limiting dilution cloning. TAP1 knockout cells were enriched by FACS sort on GFP positivity.

\section{Sanger sequencing}

Genomic DNA was isolated using the NucleoSpin Tissue kit (Machery-Nagel). DNA was amplified and sequenced using BigDye v1.1 (Applied Biosystems) and specific primers listed in Supplementary Table II.

\section{Immunoblotting}

Proteins were separated by SDS-PAGE (Bis-Tris NuPAGE 12\% gel (CALR immunoblot, 10\% Bis-Tris NuPAGE gel), ThermoFisher) and transferred to a PVDF membrane (Amersham Hybond-P, GE Healthcare) (CALR, nitrocellulose, Invitrogen) at $25 \mathrm{~V}$ for 12 min by using the semidry Trans-Blot Turbo (BioRad) system (CALR, iBlot2 semi-dry blotting system, Invitrogen). Membranes were blocked for $1 \mathrm{~h}$ with $3 \%(\mathrm{w} / \mathrm{v})$ non-fat milk powder in PBS/0.1\% (v/v) Tween 
(PBST) (CALR, 10\% Roche WBR in PBST) and incubated with primary antibody (Supplementary Table III) over night (CALR, $1 \mathrm{~h}$ ) at $4{ }^{\circ} \mathrm{C}$. After washing three times in PBST, the membrane was incubated with secondary antibody for $1 \mathrm{~h} \mathrm{RT}$ and washed again in PBST. Blots were incubated with Clarity Western ECL reagent (BioRad) (CALR, Pierce ECL plus, ThermoFisher Scientific), the signal was detected with a Lumi-Imager (Vilber, Fusion FX) (CALR, Image Lab software, Bio-Rad) and analyzed with Evolution-Capt Edge software.

Cells were incubated with specific antibodies (Supplementary Table III) diluted in PBS for 30 min on ice. Unconjugated antibodies were washed away in three times before secondary stain. Stained cells were sorted on a BD Aria II or fixed in PBS/1\% $(\mathrm{v} / \mathrm{v})$ formaldehyde $/ 1 \mu \mathrm{M}$ DAPI (Sigma-Aldrich) and analyzed by BD flow cytometers (Fortessa or LSR II). Data was analyzed using FlowJo (Tree Star, Inc). Data were gated on DAPI negativity, single cells, time and if applicable an $\triangle$ NGFR reporter.

IFN-y stimulation

Cells were cultured with $0,10,20,40$ or $80 \mathrm{U} / \mathrm{mL}$ IFN-Y (PeproTech) for 1 or $2 \mathrm{~d}$ before analysis by $\mathrm{qPCR}$ and FACS, respectively.

$q P C R$

RNA extraction and qPCR was performed as described previously(13). Gene expression was determined using SYBR green and the StepOnePlus (ThermoFisher). Quantification was done using the $\Delta \Delta \mathrm{C}_{\mathrm{T}}$ method. 18S rRNA expression was used as an internal reference, primers are listed in Supplementary Table II.

Cells were incubated for $24 \mathrm{~h}$ with $100 \mathrm{U} / \mathrm{mL}$ IFN-Y (BioLegend), pulse-chase was performed as previously described(14). Cells were metabolically labeled with $0.2 \mathrm{mCi} / \mathrm{mL}$ for $30 \mathrm{~min}$, lysed and cleared from membrane debris at $16,200 \mathrm{xg}$ for $30 \mathrm{~min}$ at $4{ }^{\circ} \mathrm{C}$. Lysates were incubated with W6/32 or MaP.ERp57 (Abcam) for $1 \mathrm{~h}$ at $4^{\circ} \mathrm{C}$ in an overhead tumbler before retrieving immune complexes with protein G Sepharose (GE Healthcare). Beads were washed and complexes were treated with Endoglycosidase $\mathrm{H}$ (New England Biolabs) according to manufacturer's protocol. Prior to loading on a 10-14\% gradient SDS-PAGE gel, complexes were dissociated at $95^{\circ} \mathrm{C}$ in sample buffer (150mM DTT).

Mass spectrometry of HLA-class I peptides. lyophilized, dissolved in 95/3/0.1\% (v/v/v) water/acetonitrile/formic acid (WAFA) and analyzed by on-line C18 nanoHPLC MS/MS with a system consisting of an Ultimate3000nano gradient 
HPLC system (Thermo, Bremen, Germany) and an Exploris480 mass spectrometer (Thermo). Fractions were injected onto a cartridge precolumn $(300 \mu \mathrm{m} \times 5 \mathrm{~mm}, \mathrm{C} 18$ PepMap, $5 \mu \mathrm{m}, 100$ A) and eluted via a homemade analytical nano-HPLC column (50 cm $\times 75 \mu \mathrm{m}$; Reprosil-Pur C18-AQ $1.9 \mu \mathrm{m}, 120 \mathrm{~A}$ (Dr. Maisch, Ammerbuch, Germany)). The gradient was run from 2\% to $36 \%(\mathrm{v} / \mathrm{v})$ solvent $B(20 / 80 / 0.1(\mathrm{v} / \mathrm{v} / \mathrm{v})$ WAFA) in $120 \mathrm{~min}$. The nano-HPLC column was drawn to a tip of $\sim 10 \mu \mathrm{m}$ and acted as the electrospray needle of the MS source. The MS was operated in data-dependent MS/MS mode for a cycle time of $3 \mathrm{~s}$, with an HCD collision energy at $30 \mathrm{~V}$ and recording of the MS2 spectrum in the orbitrap, with a quadrupole isolation width of $1.6 \mathrm{Da}$. In the master scan (MS1) the resolution was 60,000, the scan range 300-1,500, at an AGC target of $1,000,000$ at maximum fill time of $120 \mathrm{~ms}$. A lock mass correction on the background ion $m / z=445.12$ was used. Precursors were dynamically excluded after $n=1$ with an exclusion duration of $45 \mathrm{~s}$, and with a precursor range of $20 \mathrm{ppm}$. Charge states 1-3 were included. For MS2, the first mass was set to $110 \mathrm{Da}$, and the MS2 scan resolution was 30,000 at an AGC target of 100,000 at maximum fill time of $120 \mathrm{~ms}$.

Raw data were first converted to peak lists using Proteome Discoverer version 2.2 (Thermo Electron), and submitted to the Uniprot Homo sapiens minimal database (20,205 entries), using Mascot v. 2.2.04 (www.matrixscience.com) for protein identification (10 ppm precursor, $0.02 \mathrm{Da}$ deviation of fragment mass, no enzyme specified). Methionine oxidation and cysteinylation on cysteine were set as a variable modification. Peptides with an $\mathrm{FDR}<1 \%$ in combination with a mascot ion score $>35$ were accepted.

Peptide affinities were predicted using NetMHC4.0 for HLA-A*02:01, HLA-B*40:01 and HLA$C^{*} 03: 03$, which has a comparable binding motif as the expressed HLA-C*03:04(16). Peptide sequence clustering was performed for all 9-mers using GibbsCluster-2.0 and Seq2Logo.

$T$ cell assays

Target cells were incubated with $T$ cells in a 1:1 ratio for $18 \mathrm{~h}$ as previously described(17). IFN$\mathrm{Y}$ or GM-CSF release in the cell culture supernatant was measured using ELISA according to the manufacturer's protocol (Sanquin and BioLegend, respectively).

\section{Statistical analysis}

Statistical testing was done by a one-way ANOVA followed by Dunnett's multiple comparisons test (GraphPad Prism). 


\section{Results and Discussion}

We selected HAP1 as the parental cell line for creating PAKC as it has excellent cloning capacity, a high proliferation rate and a functional HLA-I antigen presentation pathway. Using CRISPR/Cas9 we created knockout cell lines for the currently known players in HLA-I antigen presentation: HLA-I heavy chain, CNX, glucosidase Ila, B2M, CALR, tapasin, ERp57, TAP1, TAP2 and ERAP1 (Figure 1A). ERAP2 and TABPBR were not targeted since RNA-seq data show that these are not expressed in HAP1 cells(18). Over time, we used different strategies to introduce the Cas 9 and gRNAs targeting the coding sequence close to the start codon of each gene to create the individual knockout cell lines. First, tapasin and ERp57 knockout cell lines were created by gRNA-directed genomic insertion of a blasticidin S-resistance gene. The second approach consisted of gRNA-driven induction of random deletions or insertions to cause frameshifts in the genes encoding HLA-I, CNX, B2M, CALR, TAP1, TAP2, ERAP1 and glucosidase Ila. HLA-I was targeted using a single gRNA recognizing a conserved sequence in the HLA-A, -B and -C genes. Of note, this gRNA is also specific for HLA-G and has one or multiple mismatches with HLA-E and HLA-F genes.

After initial selection by antibiotics or FACS sort for a reporter, clonal cell lines were generated by limiting dilution and targeted gene regions were Sanger sequenced to evaluate loss of gene integrity. We then selected knockout lines for all target genes. Notably, the most detrimental genotype for TAP1 knockout clones was a deletion of 129 bp covering the entire second transmembrane domain (Figure 1B and Supplementary Figure 1)(19). Immunoblot analysis of this clone confirmed a complete lack of TAP1 expression. Similarly, we validated the absence of tapasin, ERp57, HLA-I heavy chain, CNX and CALR proteins in the respective knockout cell lines (Figure $1 \mathrm{C}$ and $\mathrm{D}$ ).

As a final validation of PAKC, we characterized the effect of each knockout on HLA-I surface expression by flow cytometry using the HLA-I antibody W6/32 (Figure 1E and F). Except CNX and ERAP1, all APM knockouts induced a significant decrease of HLA-I surface expression confirming findings of others. ERAP1 knockout cells show a slight but significant increase in surface HLA-I, which has been observed before(20). The chaperones CALR and CNX are known to execute similar functions(21). Redundancy between these chaperones is a potential explanation for the absence of a phenotype in cells depleted of CNX. Inversely, CNX is incapable of fully substituting CALR function as HLA-I surface levels are decreased in CALR knockout cells. In depth studies into the mutual effect of these proteins on antigen presentation may thus require the additional generation of CNX and CALR double knockout cells.

We next evaluated whether PAKC provides also other opportunities to improve our understanding of HLA-I antigen presentation. In fact, our straightforward immunoblot analyses 
indicated that the knockout of TAP1, HLA-I, B2M or ERAP1 decreased tapasin expression, suggesting a novel role for each of these in stabilizing tapasin (Figure 1C). This is relevant to the ongoing debate whether the protease ERAP1 is accommodated within the peptide loading complex(22). The regulation of tapasin homeostasis and the functional consequences thereof are still unknown. In the opposite direction, tapasin has been described to stabilize TAP, showing the complexity of peptide loading complex regulation(23). These findings demonstrate that PAKC provides opportunities to understand the interplay between different APM components.

HLA-I is highly polymorphic with more than 17,000 different alleles identified in the human population. Several of these alleles and other APM polymorphisms have been associated with susceptibility or resistance to infection, the development of cancer and autoimmunity(1). To investigate the feasibility of studying such disease-associated variations with PAKC, we reconstituted a set of ten HLA-I alleles into wild type HAP1 cells. These alleles were individually detected using allele specific antibodies that do not cross-react with the endogenous HLA-I alleles (Figure 2A). Thus, PAKC can be employed to investigate the properties of individual HLA-I alleles in the context of other (dys)functional APM component, which will improve our understanding of the association of the antigen presentation pathway with HLA-I associated diseases.

After initial synthesis of the HLA-I heavy chain, the molecule matures through a process of folding, peptide loading and glycosylation events. We investigated whether PAKC allows for evaluation of individual APM component contributions to HLA-I maturation using pulse chase experiments. In wild type cells, ERp57 interacted only transiently with pulsed HLA-I, indicating that the HLA-I peptide loading largely occurred within 45 minutes (Figure 2B). The majority of HLA-I further matured within 90 minutes after pulse as detected by EndoH resistance of the $N$ glycan of immunoprecipitated HLA-I (Figure 2C). Such molecular studies will further catalyze new mechanistic and disease-related insights after overexpression of individual HLA-I alleles or mutant APM members in their respective knockout cells.

After two decades of algorithm development to predict the immunogenic HLA-I presented peptide repertoire, the scientific community is still facing major issues in the accuracy of these predictions. The contribution of several APM members to peptide selection has not been interrogated, mainly due to the lack of proper model cell lines. Although HAP1 cells have a lower HLA-I surface expression than the commonly used JY cell line, we investigated whether PAKC could be employed for HLA-I ligandome analyses on HAP1 wild type cells. Mass spectrometric analyses of eluted peptides identified 3774 peptides that were assigned to bind HLA-A*02:01, HLA-B*40:01 or HLA-C*03:04 based on peptide affinity prediction (Figure 2D). 
Sequence logos corresponding to the 9-mer peptides for HLA-A*02:01, HLA-B*40:01 and HLA$C^{*}$ 03:04 were in concordance with literature (Figure 2E) $(16,24,25)$. Thus, PAKC is well-suited for high-resolution peptide elution studies which further benefit from the fact that PAKC is made on a single genetic and proteomic background. This property can be further exploited by studies to individual peptide processing and presentation which, besides targeted mass spectrometry, may be read out by peptide-specific $T$ cell activation assays. This was confirmed by the cytokine production of two independent $T$ cell clones recognizing endogenously derived peptides presented by HLA-A*02:01 on HAP1 wild type but not on control HLA-I knockout cells Figure 2F). Proteases other than ERAP1 described to affect peptide generation, including the proteasome, potentially have a profound effect on processing and presentation of individual peptides. These have not been included in the current PAKC, but it would be very useful to additionally include individual protease knockouts in the future. Thus, PAKC will contribute to a better understanding of the processing and HLA-I loading of peptides. The potential improvements of HLA-I ligandome prediction algorithms will advance translational research to infection, cancer and autoimmunity.

During infection, cancer and autoimmunity, antigen presentation is enhanced by locally produced cytokines including IFN-y. The IFN-y signaling pathway is intact in HAP1 cells(26). Our experiments now showed that induction of IFN-y signaling upregulates HLA-I mRNA and surface protein levels (Figure $2 \mathrm{E}$ and $\mathrm{F}$ ). These findings demonstrate that PAKC is highly suitable to study antigen presentation in an inflammatory context, for example in any of the above-mentioned research directions. Additionally, PAKC also supports research to nonclassical HLA-I molecules, which are lowly expressed on HAP1 cells, but are expressed upon IFN-y stimulation and exogenous peptide feeding. Finally, the haploid nature of HAP1 cells, and most of our clones, allows for powerful genome-wide haploid genetic screening to unravel novel antigen presentation biology(27).

In conclusion, we generated and validated a novel panel of ten cell lines, each knockout for an individual APM component which will accelerate antigen presentation research. We showed that this panel can be adopted into many different research lines as it is suitable for a wide variety of assays. As pointed out, PAKC will expand our knowledge by answering open questions in the field for which no good model systems were previously available(28). It will facilitate generation of new hypotheses, as well as revisiting and challenging published data that were generated in imperfect or inconsistent model systems over the years. PAKC is now available for the scientific community to enable unraveling of novel antigen presentation biology, some of which eventually may lead to new therapeutic approaches to treat infections, cancer and autoimmunity. 


\section{Acknowledgments}

290 We thank Dr. Ann-Charlott Schneider for performing qPCR and the Sanquin Research Facility 291 for their assistance with FACS. Furthermore, we thank Dr. Sebastian Springer and Dr. Zeynep

292 Hein (Jacobs University) for providing the HLA-B`27:05 and HLA-B*27:09 overexpression 293 constructs, Dr. Marieke Griffioen (LUMC) for providing the SSR1 specific T cell clone, all other 294 HLA-I overexpression constructs, as well as critically reading the manuscript, Dr. Mirjam 295 Heemskerk (LUMC) for providing the USP11 specific T cell clone, Dr. Riccardo Mezzadra (The 296 Netherlands Cancer Institute) for the B2M CRISPR/Cas9 construct and Dr. Jacques Neefjes (LUMC) for providing the HC10 antibodies. 


\section{References}

299

300

301

302

303

304

305

306

307

308

309

310

311

312

313

314

315

316

317

318

319

320

321

322

323

1. McGonagle, D., S. Z. Aydin, A. Gül, A. Mahr, and H. Direskeneli. 2015. 'MHC-I-opathy'unified concept for spondyloarthritis and Behçet disease. Nat. Rev. Rheumatol. 11: 731-40.

2. Halenius, A., C. Gerke, and H. Hengel. 2015. Classical and non-classical MHC i molecule manipulation by human cytomegalovirus: So many targets - But how many arrows in the quiver? Cell. Mol. Immunol. 12: 139-153.

3. Neefjes, J., M. L. M. M. Jongsma, P. Paul, and O. Bakke. 2011. Towards a systems understanding of MHC class i and MHC class II antigen presentation. Nat. Rev. Immunol. 11: 823-836.

4. Gettinger, S., J. Choi, K. Hastings, A. Truini, I. Datar, R. Sowell, A. Wurtz, W. Dong, G. Cai, M. A. Melnick, V. Y. Du, J. Schlessinger, S. B. Goldberg, A. Chiang, M. F. Sanmamed, I. Melero, J. Agorreta, L. M. Montuenga, R. Lifton, S. Ferrone, P. Kavathas, D. L. Rimm, S. M. Kaech, K. Schalper, R. S. Herbst, and K. Politi. 2017. Impaired HLA class I antigen processing and presentation as a mechanism of acquired resistance to immune checkpoint inhibitors in lung cancer. Cancer Discov. 7: 1420-1435.

5. Blees, A., D. Januliene, T. Hofmann, N. Koller, C. Schmidt, S. Trowitzsch, A. Moeller, and R. Tampé. 2017. Structure of the human MHC-I peptide-loading complex. Nature 551: 525528.

6. Trowitzsch, S., and R. Tampé. 2019. Multifunctional chaperone and quality control complexes in adpative immunity. Annu. Rev. Biophys. .

7. Amir, A. L., D. M. van der Steen, R. S. Hagedoorn, M. G. D. Kester, C. A. M. van Bergen, J. W. Drijfhout, A. H. de Ru, J. H. F. Falkenburg, P. A. van Veelen, and M. H. M. Heemskerk. 2011. Allo-HLA-reactive T cells inducing graft-versus-host disease are single peptide specific. Blood 118: 6733-6742.

8. Van Bergen, C. A. M., C. E. Rutten, E. D. Van Der Meijden, S. A. P. Van Luxemburg-Heijs, E. G. A. Lurvink, J. J. Houwing-Duistermaat, M. G. D. Kester, A. Mulder, R. Willemze, J. H. F. Falkenburg, and M. Griffioen. 2010. High-Throughput Characterization of 10 New Minor Histocompatibility Antigens by Whole Genome Association Scanning. Cancer Res. 70: 90739083.

9. Spaapen, R. M., R. W. J. Groen, K. van den Oudenalder, T. Guichelaar, M. van Elk, T. Aarts-Riemens, A. C. Bloem, G. Storm, A. C. Martens, H. M. Lokhorst, and T. Mutis. 2010. Eradication of Medullary Multiple Myeloma by CD4+ Cytotoxic Human T Lymphocytes Directed at a Single Minor Histocompatibility Antigen. Clin. Cancer Res. 16: 5481-5488. 
10. Lackner, D. H., A. Carré, P. M. Guzzardo, C. Banning, R. Mangena, T. Henley, S. A generic strategy for CRISPR-Cas9-mediated gene tagging. Nat. Commun. 6: 10237. survey the structural integrity of $H L A-B^{\star} 27: 05$ intracellularly and at the surface. PLoS One 13: e0200811.

12. van Bergen, C. A. M., S. A. P. van Luxemburg-Heijs, L. C. de Wreede, M. Eefting, P. A. von dem Borne, P. van Balen, M. H. M. Heemskerk, A. Mulder, F. H. J. Claas, M. A. Navarrete, W. M. Honders, C. E. Rutten, H. Veelken, I. Jedema, C. J. M. Halkes, M. Griffioen, and J. H. F. Falkenburg. 2017. Selective graft-versus-leukemia depends on magnitude and diversity of the alloreactive T cell response. J. Clin. Invest. 127: 517-529. Cytokines and Growth Factors in Oesophageal Adenocarcinoma Reveal a Tumour Immune Escape-promoting Microenvironment. Scand. J. Immunol. 68: 616-623. Schwarzmüller, V. T. K. Le-Trilling, S. Stevanovic, H. Hengel, F. Momburg, and A. Halenius. 2019. HLA-B locus products resist degradation by the human cytomegalovirus immunoevasin US11. PLOS Pathog. 15: e1008040.

15. van der Lee, D. I., R. M. Reijmers, M. W. Honders, R. S. Hagedoorn, R. C. de Jong, M. G. Kester, D. M. van der Steen, A. H. de Ru, C. Kweekel, H. M. Bijen, I. Jedema, H. Veelken, P. A. van Veelen, M. H. Heemskerk, J. H. F. Falkenburg, and M. Griffioen. 2019. Mutated nucleophosmin 1 as immunotherapy target in acute myeloid leukemia. J. Clin. Invest. 129: 774-785.

16. Rasmussen, M., M. Harndahl, A. Stryhn, R. Boucherma, L. L. Nielsen, F. A. Lemonnier, M. Nielsen, and S. Buus. 2014. Uncovering the Peptide-Binding Specificities of HLA-C: A General Strategy To Determine the Specificity of Any MHC Class I Molecule. J. Immunol. 193: 4790-4802.

17. Spaapen, R. M., H. M. Lokhorst, K. van den Oudenalder, B. E. Otterud, H. Dolstra, M. F. Leppert, M. C. Minnema, A. C. Bloem, and T. Mutis. 2008. Toward targeting B cell cancers with CD4+ CTLs: identification of a CD19-encoded minor histocompatibility antigen using a novel genome-wide analysis. J. Exp. Med. 205: 2863-2872. 
Reveals Fibroblast Growth Factors as Novel Inhibitors of Viral Replication. J. Virol. 92.

19. Koch, J., R. Guntrum, S. Heintke, C. Kyritsis, and R. Tampé. 2004. Functional Dissection of the Transmembrane Domains of the Transporter Associated with Antigen Processing (TAP). J. Biol. Chem. 279: 10142-10147.

20. Tran, T. M., S. Hong, J. H. Edwan, and R. A. Colbert. 2016. ERAP1 reduces accumulation of aberrant and disulfide-linked forms of HLA-B27 on the cell surface. Mol. Immunol. 74: 10-17.

21. Danilczyk, U. G., M. F. Cohen-Doyle, and D. B. Williams. 2000. Functional Relationship between Calreticulin, Calnexin, and the Endoplasmic Reticulum Luminal Domain of Calnexin. J. Biol. Chem. 275: 13089-13097.

22. Chen, H., L. Li, M. Weimershaus, I. Evnouchidou, P. van Endert, and M. Bouvier. 2016. ERAP1-ERAP2 dimers trim MHC I-bound precursor peptides; implications for understanding peptide editing. Sci. Rep. 6: 28902.

23. Blees, A., K. Reichel, S. Trowitzsch, O. Fisette, C. Bock, R. Abele, G. Hummer, L. V. Schäfer, and R. Tampé. 2015. Assembly of the MHC I peptide-loading complex determined by a conserved ionic lock-switch. Sci. Rep. 5: 17341.

24. Andreatta, M., O. Lund, and M. Nielsen. 2013. Simultaneous alignment and clustering of peptide data using a Gibbs sampling approach. Bioinformatics 29: 8-14.

25. Yarzabek, B., A. J. Zaitouna, E. Olson, G. N. Silva, J. Geng, A. Geretz, R. Thomas, S. Krishnakumar, D. S. Ramon, and M. Raghavan. 2018. Variations in HLA-B cell surface expression, half-life and extracellular antigen receptivity. Elife 7.

26. Mezzadra, R., M. de Bruijn, L. T. Jae, R. Gomez-Eerland, A. Duursma, F. A. Scheeren, T. R. Brummelkamp, and T. N. Schumacher. 2019. SLFN11 can sensitize tumor cells towards IFN-y-mediated T cell killing. PLoS One 14: e0212053.

27. Brockmann, M., V. A. Blomen, J. Nieuwenhuis, E. Stickel, M. Raaben, O. B. Bleijerveld, A. F. M. Altelaar, L. T. Jae, and T. R. Brummelkamp. 2017. Genetic wiring maps of singlecell protein states reveal an off-switch for GPCR signalling. Nature 546: 307-311.

28. Rock, K. L., E. Reits, and J. Neefjes. 2016. Present Yourself! By MHC Class I and MHC Class II Molecules. Trends Immunol. 37: 724-737. 
394 Figure 1. Validation of PAKC on sequence and protein level.

395 (A) Schematic overview of the HLA-I antigen presentation pathway. (B) Sequence summary 396 of one selected clone per targeted APM component. Shown are CRISPR/Cas9 induced 397 mutations (dashes), the gRNA (red) and PAM sequence (green). Tapasin ERp57 knockout 398 clones were generated by genomic insertion of a blasticidin S-resistance gene (underlined). 399 Chr, chromosome. Raw data in Supplementary Figure 1. (C), (D) Immunoblots of knockout cell 400 lines with indicated antibodies. (E), (F) FACS analysis of indicated knockout cells using the 401 pan-HLA-I antibody W6/32. (E) Representative plots with wild type in blue, knockout in red and 402 unstained control in grey. (F) Relative quantification, $n=6$ independent experiments (TAP2 403 knockout $n=3$ ). One-Way ANOVA before normalization, ${ }^{*} p<0.05,{ }^{* *} p<0.01,{ }^{* * *} p<0.0001$, 404 ns=not significant. WT, wild type; KO, knockout. 
Figure 2. Research directions of PAKC.

406 (A) HAP1 wild type cells transduced with different HLA-A and -B alleles and stained using 407 indicated allele-specific antibodies (red), untransduced cells (grey). Gated on marker gene 408 positive cells. (B), (C) Analysis of the HLA-I maturation by pulse-chase in IFN-y stimulated 409 HAP1 wild type and HLA-I knockout cells metabolically labeled with [ $\left.{ }^{35} \mathrm{~S}\right]-\mathrm{Met} / \mathrm{Cys}$ for $30 \mathrm{~min}$ 410 and chased for 0,45 or $90 \mathrm{~min}$. (B) Co-immunoprecipitation of HLA-I using anti-ERp57 411 antibodies was visualized using phospho-imaging. (C) Co-immunoprecipitation using W6/32 412 for HLA-I followed or not by EndoH treatment. (D) 3,774 unique peptides were eluted from 413 HAP1 wild type cells as identified by mass spectrometry and allocated to the allele with the 414 highest predicted affinity using NetMHC4.0 with an affinity cutoff of $5 \mu \mathrm{M}$. (E) All identified 9415 mer peptides were used to perform unsupervised sequence clustering and visualized by 416 Seq2Logo to reveal common sequences for each HLA-I allele. (F) IFN-y or GM-CSF secretion 417 by $\mathrm{HLA}-\mathrm{A}^{*} 02: 01$-restricted $\mathrm{T}$ cells specific for USP11- or SSR1-derived peptides after 418 overnight co-culture with HAP1 wild type or HLA-I knockout cells. (G) Upregulation of HLA-I 419 mRNA and $(\mathbf{H})$ cell surface HLA-I after IFN-y stimulation as assessed by qPCR and FACS, 420 respectively. WT, wild type; $\mathrm{KO}$, knockout. 
FiguręXiv preprint doi: https://doi.org/10.1101/2020.01.24.917807; this version posted January 25, 2020. The copyright holder for this preprint (which was not certified by peer review) is the author/fund r r

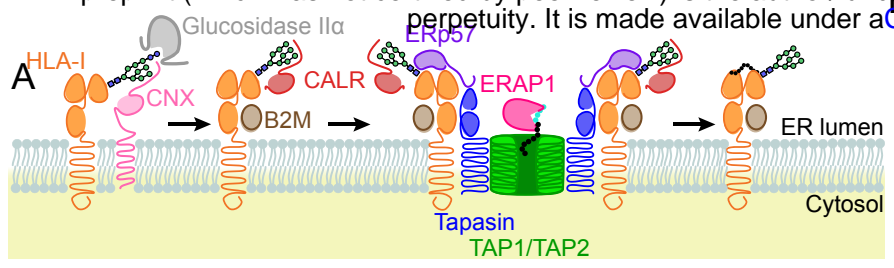

C
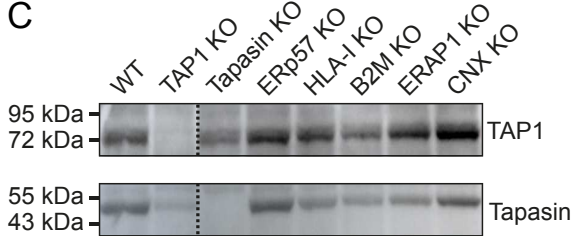

$51 \mathrm{kDa}$

D
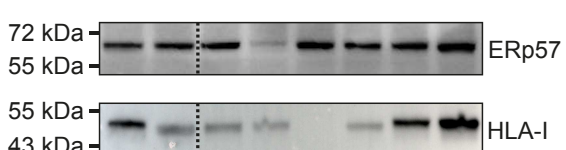

$95 \mathrm{kDa}-\longrightarrow \mathrm{kDa}-\longrightarrow-\ldots$

$55 \mathrm{kDa}-$

$43 \mathrm{kDa}$

$39 \mathrm{kDa}$

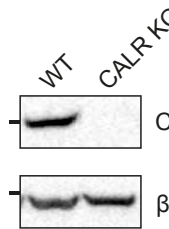

20

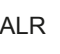

$\underline{\text { CALR KO }}$

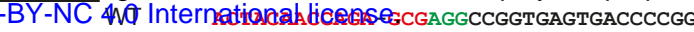

HLA-A KO ACTACAACCAGAAGCGAGGCCGGTGAGTGACCCCGG +1bp

HLA-B KO ACTACAACCAGAAGCGAGGCGGTGAGTGACCCCG +1bp

HLA-C KO ACTACAACCAGAAGCGAGGCCGGTGGTGACCCCGG +1bp

CNXKO

WT GGAAGTGGTTGCTGTGTATGTT-ACTGGTGCTTGGA

KO chr A GGAAGTGGTTGCTGTGTATGTTTACTGGTGCTTGGA +1bp

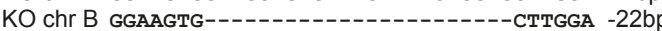

Glucosidase lla KO

WT AACAGTGTGGAGTTAACCATGGCTGAGGGACCCTAC

B2M KO

KO AACAGTGTGGAG-----.----ACCCTAC -17bp

WT ACTCCAAAGATTCAGG-TTTACTCACGTCATCCAGC

KO chr A ACTCCAAAGATTCAGGATTTACTCACGTCATCCAGC +1bp

KO chr B АCTCCA-----_---_----GTCATCCAGC -19bp

WT ACGGGTGGACTTCCCGCTGGATCGAATCCAAACACA

KO ACGGGTGGACTT---------CGAATCCAAACACA -10bp

Tapasin KO

Actin $\frac{\text { GA }}{W}$ GAGGATGGAGCGGAAAGGGCCTGGCCAAGAGACCC

ERp57 KO

GAGGATGCGAGCGTCCAGGGTGGAAGCGGAGCTACT Blast ins

WT GCTGCCTCCGACGTGCTAGAACTCACGGACGACAAC

KO GCTGCCTCCGACGTCCAGGGTGGAAGCGGAGCTACT Blast ins

WT AGTCCCAGGCCC/129bP/GA--CTGGGTGCTGCTC

KO AGTCCCAGGCCC/-----/--TGCTGGGTGCTGCTC -129bp

TAP2 KO

WT GGGTACGGGGCTGCGGGGTCAGCTAGTCAGTGTGG

ERAP1 KO

GGGTACGGGGCT--------CAGCTAGTCAGTGTGG -8bp

TGAAGCATCTCCAAA-ACGTAGTGATGGGACACCAT

KO chr A TGAAGCATCTCCAAAAACGTAGTGATGGGACACCAT +1bp

- WT - KO - Unstained

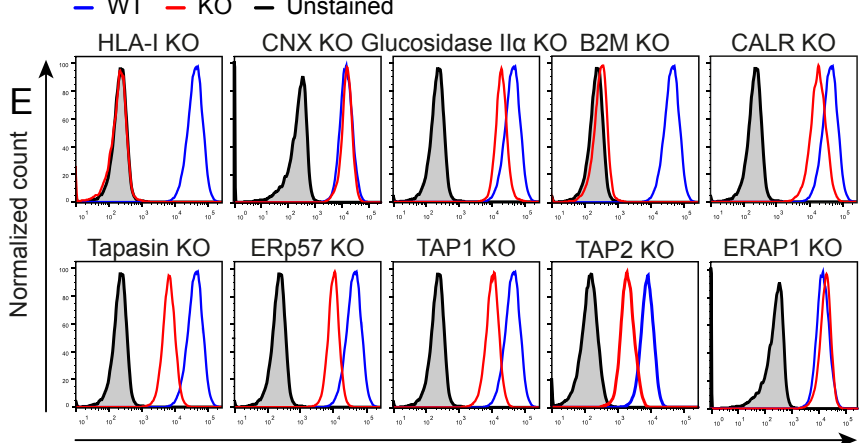

F

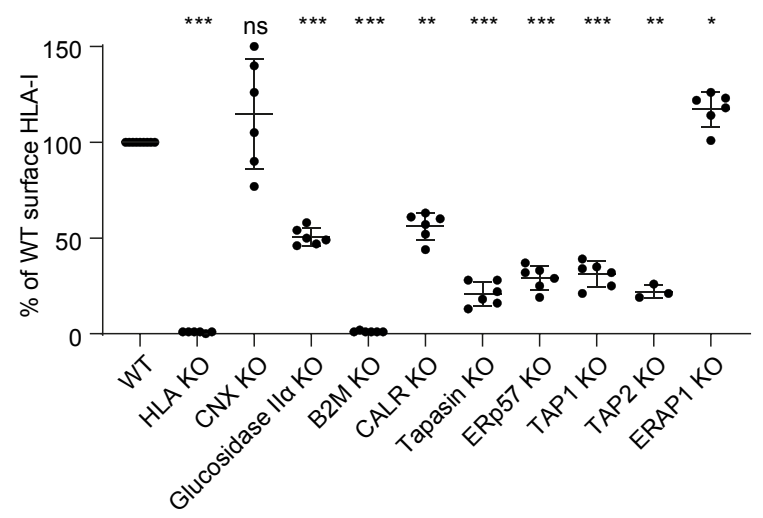


Figutaxiv preprint doi: https://doi.org/10.1101/2020.01.24.917807; this version posted January 25, 2020. The copyright holder for this preprint (which was not certified by peer review) is the author/funder, who has granted bioRxiv a license to display the preprint in fransduced WT - Untransqfeegsdettlity. It is made available under aCC-BY-NC 4.0 International license.
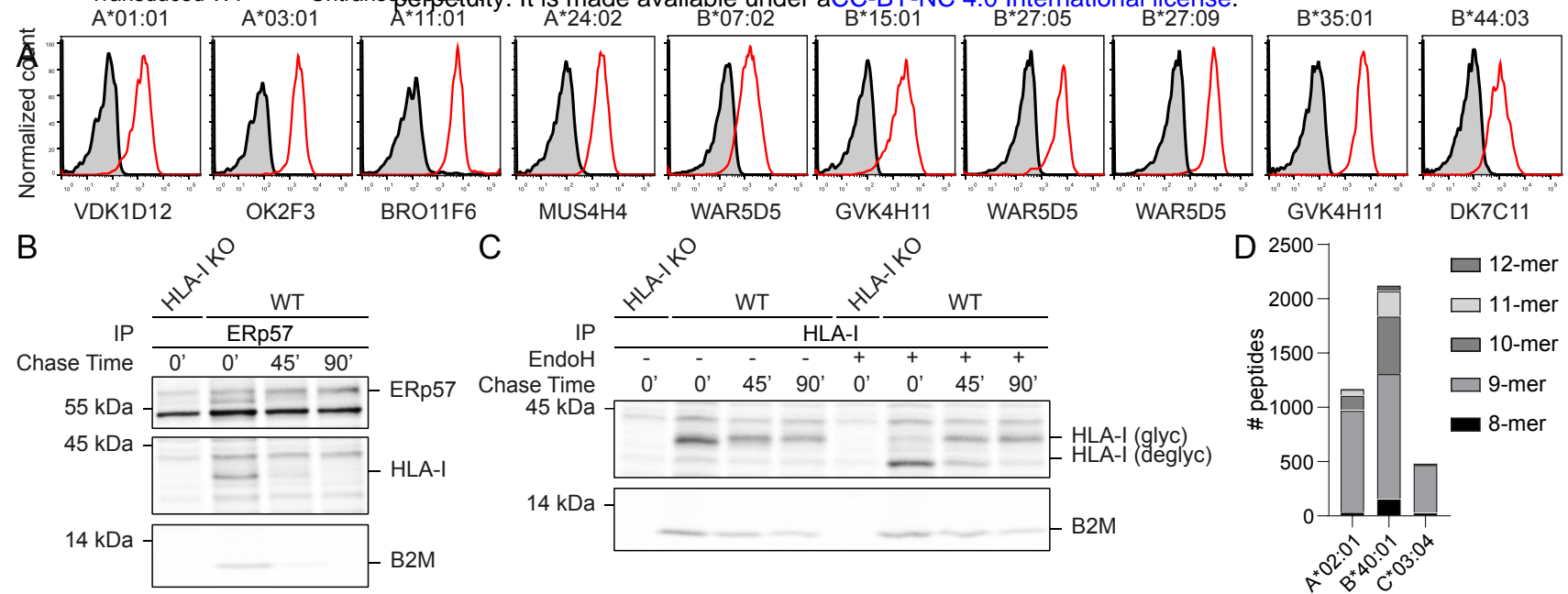

C

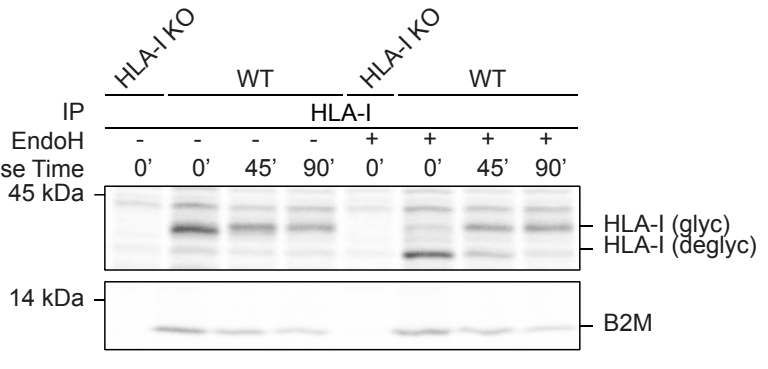

D 2500

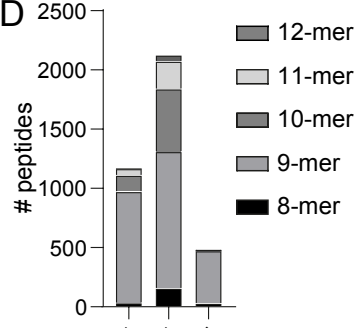

$\mathrm{F}$

G

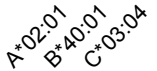

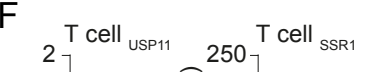

$\mathrm{H}-$ Unstimulated

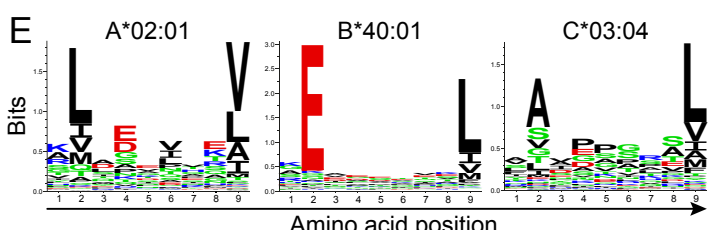

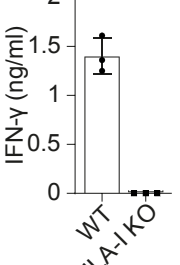
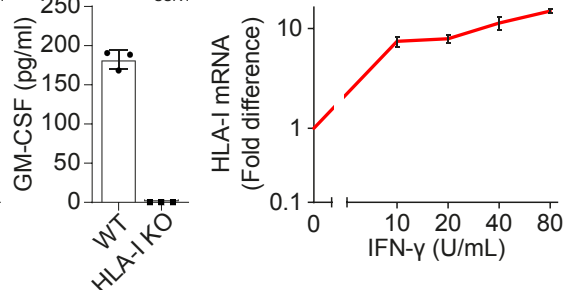

$-20 \mathrm{U} / \mathrm{mL}$ IFN-Y - Unstained

으. W6/32 (HLA-I) 


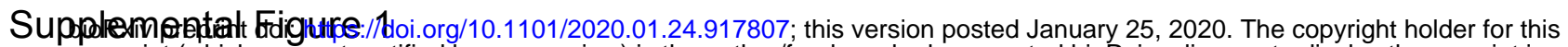
preprint (which was not certified by peer review) is the author/funder, who has granted bioRxiv a license to display the preprint in

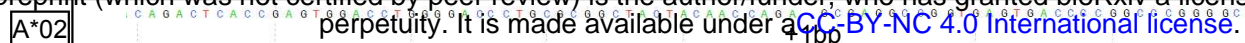

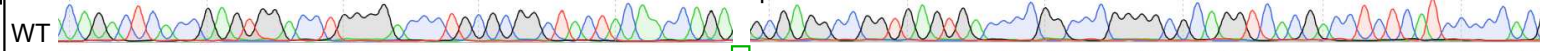

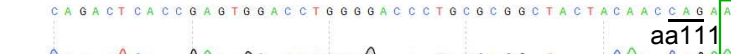

$\mathrm{B}^{*} 40$

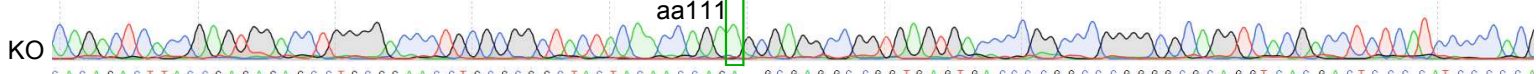

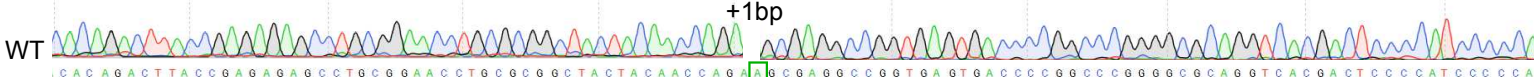
HLA-I TKO

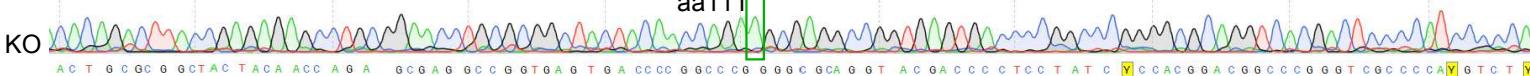

$\mathrm{C}^{*} 03$

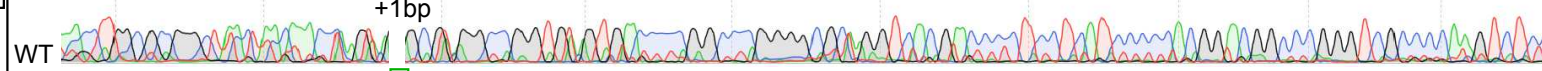
aa111

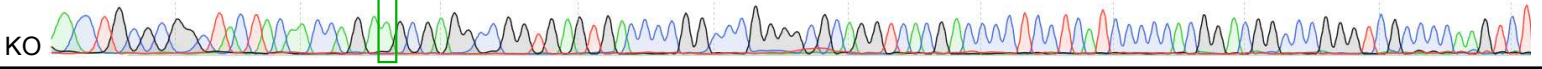
Chromosome A

Chromosome B

\begin{tabular}{rl|}
\hline aa9 +1 \\
\hline aa4 \\
\hline$-22 \mathrm{bp}$
\end{tabular}

Frameshift

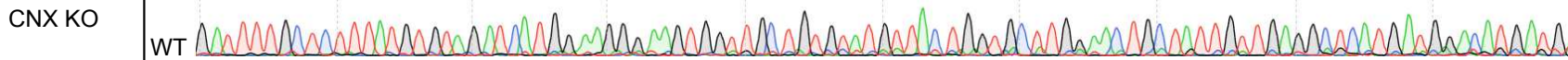

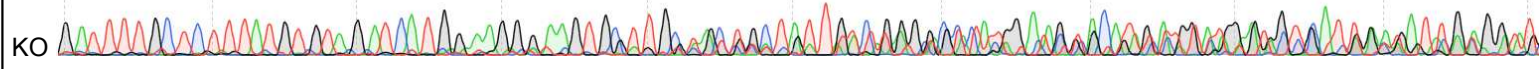

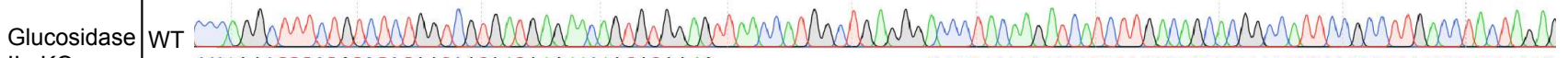
lla KO

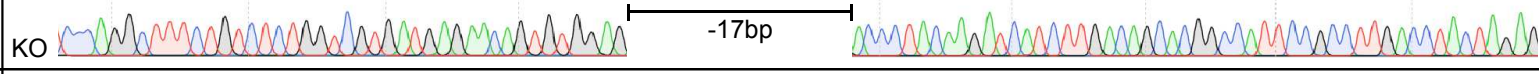

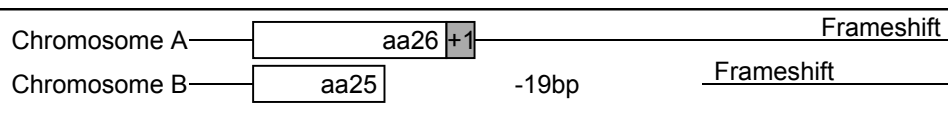

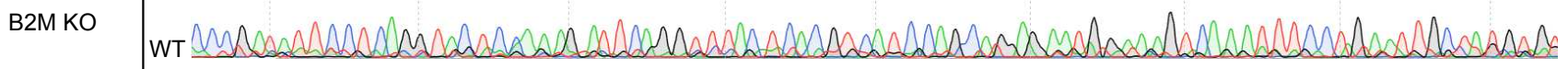

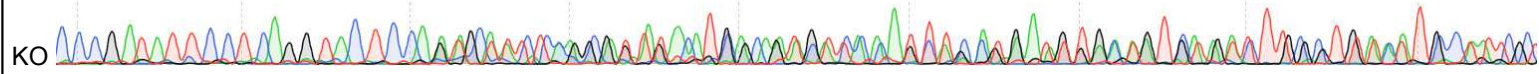
$\frac{\mathrm{ACCT}}{\mathrm{a} a 34}$

CALR KO

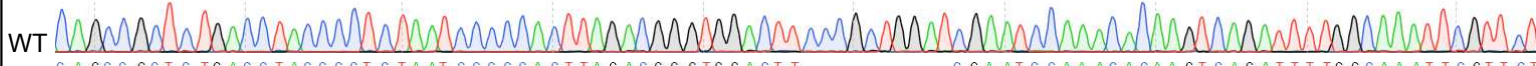

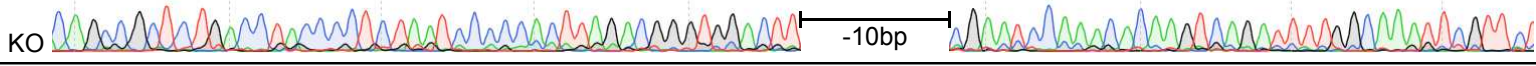

Tapasin KO

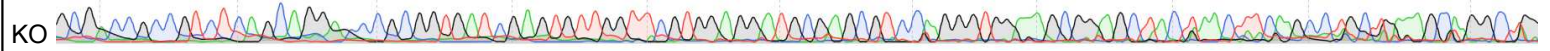

ERp57 KO

aa26 Blasticidin resistance gene

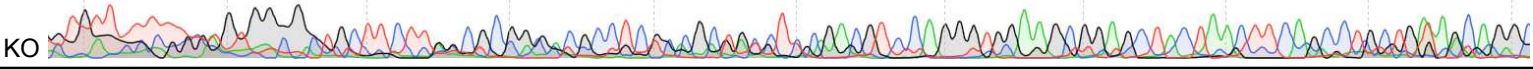
$-131 \mathrm{bp}$

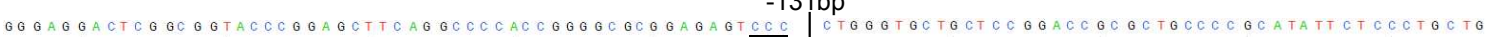

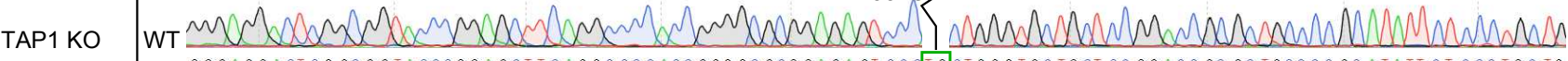

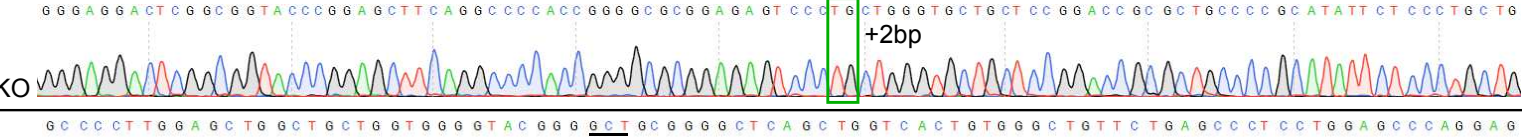
aล108

TAP2 KO

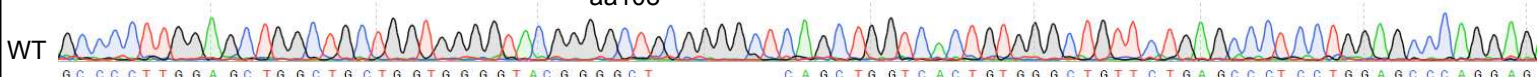

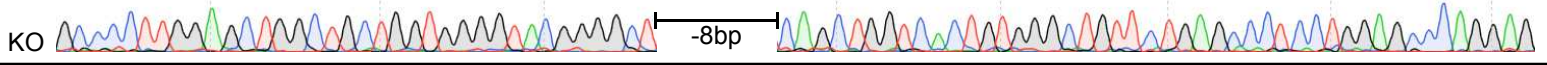

\begin{tabular}{|c|c|}
\hline Chromosome A & aa40 +1 Frameshift \\
\hline Chromosome B & Frameshift \\
\hline
\end{tabular}

ERAP1 KO

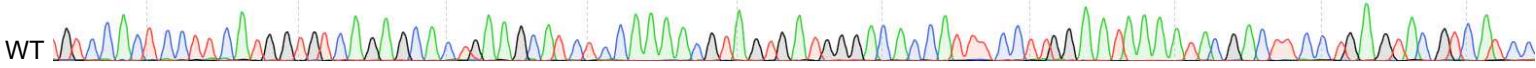

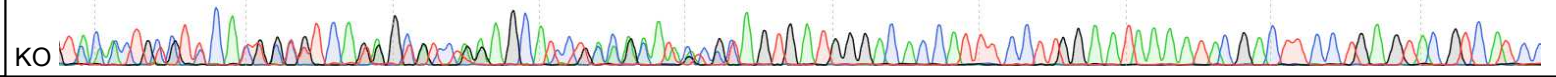


Supplemental Figure 1. Sequencing details of PAKC.

Sanger sequences around the Cas9 targeted site for each clonal cell line in PAKC. For each clone, wild type and knockout sequences are shown. For cases of blasticidin S-resistance gene insertion or mixed sequences due to diploidy of the cells, a graphical summary is shown above the sequences (upper sequence annotation refers to chromosome $A$ and lower to chromosome B). The number of amino acids (aa) translated before the disrupting mutation are shown, underlined are translation start sites, green squares highlight base pair insertions. WT, wild type; KO, knockout. 


\section{Supplemental Table I. Summary of clonal cell lines of PAKC}

\begin{tabular}{|c|c|c|c|c|c|}
\hline $\begin{array}{l}\text { Targeted } \\
\text { gene(s) }\end{array}$ & gRNA & Vector used & Resistance & $\begin{array}{l}\text { Clone } \\
\text { name }\end{array}$ & $\begin{array}{l}\text { Intact aa I } \\
\text { (total aa) }\end{array}$ \\
\hline $\begin{array}{l}\text { HLA-A*02:01 } \\
\text { HLA-B*40:01 } \\
\text { HLA-C*03:04 }\end{array}$ & CGGCTACTACAACCAGAGCG & pX330 & puromycin & $\# 66$ & $\begin{array}{l}111(365) \\
111(362) \\
111(366)\end{array}$ \\
\hline CNX & GTGGTTGCTGTGTATGTTAC & pLentiCRISPRv2 & puromycin & \#3F5 & $9(592)$ \\
\hline Glucosidase Ila & GAACAGTGTGGAGTTAACCA & pLentiCRISPRv2 & puromycin & \#A10 & $139(966)$ \\
\hline B2M & CGTGAGTAAACCTGAATCTT & $\mathrm{pX} 458$ & none & $\# 14.2$ & $26(119)$ \\
\hline CALR & GTGTTTGGATTCGATCCAGC & pLentiCRISPRv2 & puromycin & \#1F9 & $34(417)$ \\
\hline Tapasin & CGTGGAGGATGCGAGCGGAA & $\mathrm{p} \times 330$ & blasticidin $\mathrm{S}$ & \#29 & $34(448)$ \\
\hline ERp57 & GTCCGTGAGTTCTAGCACGT & $\mathrm{pX330}$ & blasticidin S & \#B & $26(505)$ \\
\hline TAP1 & ACTGCTACTTCTCGCCGACT & pL.CRISPR.efs.GFP & none & \#1 & $\begin{array}{l}48 / 91-808 \\
(808)\end{array}$ \\
\hline TAP2 & CTGGTGGGGTACGGGGCTGC & pLentiCRISPRv2 & puromycin & \#18 & $108(686)$ \\
\hline ERAP1 & GGTGTCCCATCACTACGTTT & pLentiCRISPRv2 & puromycin & \#2C6 & $40(941)$ \\
\hline
\end{tabular}




\section{Supplemental Table II. Primers used for PCR, qPCR and Sequencing}

\begin{tabular}{|c|c|c|c|}
\hline Targeted gene & Forward primer & Reverse primer & Sequencing primer \\
\hline HLA-A & TTCTTCACATCCGTGTCCCG & TTCСTCTCССTCAGGACCAG & TCCСАATTGTСTССССТССТ \\
\hline HLA-B & CCGGGAGACACAGATCTCCA & CAGCTTGTCCTTCCCGTTCT & GAGCCACTCCACGCACTC \\
\hline HLA-C & АCTTCATCGCAGTGGGCTAC & GGATCTCAGACCGGGAGACT & ACACAGAAGTACAAGCGCCA \\
\hline CNX & СTCTAGGCTGCCTTTCTTTATCT & GCACCCGGGTATCTTCATAA & AAGTTCTTTTTAGCTCTGCGATTT \\
\hline Glucosidase Ila & ATGCTTGGGTCTGTTTCTGG & GCССTCTGATGCTCAAACTC & TTCCCCCGGTCTTCTAAAGT \\
\hline B2M & GGGAGAAATCGATGACCAAA & ATGTATTTGTGCAAGTGCTGC & GGGAGAAATCGATGACCAAA \\
\hline CALR & CCGAGGATCTCTGAAGGCAC & GTTTCACCGTGAACTGCACC & GCCGACAGAGCATAAAAGCG \\
\hline Tapasin & TCGCCCAAGAACTAGAGGGA & CGACATGGTGCTTGTTGTCC & TGGTGATCTTCTCAGTGGCG \\
\hline ERp57 & AATCGGGCAATGAGGTGGAG & GAATGAGAAATGTGCCCGCC & GAATGAGAAATGTGCCCGCC \\
\hline TAP1 & CAGCCTGTTCCTGGGACTTT & ACTGACAACGAAGGCGGTAG & GCTCCCCATGAGATCAGCTC \\
\hline TAP2 & GCCTGAAAGGGCCTAGAAAT & ATTTGTGGGGACACTGCTG & TGGAGTTAGGGAAGTGAAGACC \\
\hline ERAP1 & СТСТСTCСAGСTСССTTССТ & GTGTTTCTGCCССTCAAATG & TACTTTCGTGGTTCCCCAGA \\
\hline HLA-I qPCR & TCTCCAGAAGGCACCACCA & TTCTACCCTGCGGAGATCACA & - \\
\hline 18S qPCR & CGGCTACCACATCCAAGGAA & GCTGGAATTACCGCGGCT & - \\
\hline
\end{tabular}


Supplemental Table III. Antibody clones used for immunoblotting and flow cytometry

\begin{tabular}{|c|c|c|c|}
\hline Antibody & Species & Used to Target & Manufacturer \\
\hline Calnexin polyclonal & Rabbit & CNX & $\begin{array}{l}\text { Stressgen } \\
\text { Biotechnologies } \\
\text { (SPA-865) }\end{array}$ \\
\hline Calreticulin polyclonal & Rabbit & CALR & Sigma (C4606) \\
\hline $\mathrm{HC} 10$ & Mouse & HLA-I & $\begin{array}{l}\text { Kindly provided by } \\
\text { Dr. J. Neefjes }\end{array}$ \\
\hline MaP.Erp57 & Mouse & ERp57 & Abcam \\
\hline PaSta1 & Rat & tapasin & Self \\
\hline 148.3 & Mouse & TAP1 & Self \\
\hline AC-74 & Mouse & $\beta$-actin & Sigma-Aldrich \\
\hline Anti-Mouse-HRP polyclonal & Goat & Mouse antibody & Sigma-Aldrich \\
\hline Anti-Rabbit-HRP polyclonal & Goat & Rabbit antibody & Sigma-Aldrich \\
\hline Anti-Rat-HRP polyclonal & Goat & Rat antibody & Sigma-Aldrich \\
\hline W6/32-PerCP-eFluor710 & Mouse, IgG & HLA-A, -B, -C & eBioscience \\
\hline VDK1D12 & Human, IgM & $\mathrm{HLA}^{-\mathrm{A}^{*} 01: 01}$ & Self \\
\hline OK2F3 & Human, IgM & HLA-A*03:01 & Self \\
\hline BRO11F6 & Human, IgG & HLA-A $^{*} 11: 01$ & Self \\
\hline MUS4H4 & Human, IgG & HLA-A $^{\star} 24: 02$ & Self \\
\hline WAR5D5 & Human, IgG & $\begin{array}{l}\text { HLA-B }^{*} 07: 02 \\
\text { HLA-B*2 } 27: 05 \\
\text { HLA-B* } 27: 09\end{array}$ & Self \\
\hline GVK4H11 & Human, IgM & $\begin{array}{l}\text { HLA-B*15:01 } \\
\text { HLA-B*35:01 }\end{array}$ & Self \\
\hline DK7C11 & Human, IgG & HLA-B*44:03 & Self \\
\hline MH16-1 in-house conjugated to DL650 & Mouse, IgG & Human IgG & Sanquin \\
\hline MHM-88-APC & Mouse, IgG & Human IgM & BioLegend \\
\hline ME20.4-PECy7 & Mouse, IgG & Human NGFR & BioLegend \\
\hline
\end{tabular}

